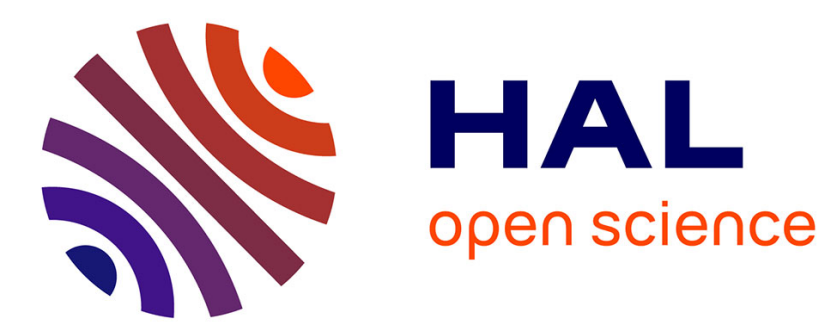

\title{
UN MALENTENDU A PROPOS DE LA FORMULE DE FLEISCHMANN
}

L. Panchaud

\section{To cite this version:}

L. Panchaud. UN MALENTENDU A PROPOS DE LA FORMULE DE FLEISCHMANN. Le Lait, 1923, 3 (10), pp.777-781. hal-00894719

\section{HAL Id: hal-00894719 \\ https://hal.science/hal-00894719}

Submitted on 1 Jan 1923

HAL is a multi-disciplinary open access archive for the deposit and dissemination of scientific research documents, whether they are published or not. The documents may come from teaching and research institutions in France or abroad, or from public or private research centers.
L'archive ouverte pluridisciplinaire HAL, est destinée au dépôt et à la diffusion de documents scientifiques de niveau recherche, publiés ou non, émanant des établissements d'enseignement et de recherche français ou étrangers, des laboratoires publics ou privés. 
rée, en employant une pipette divisée en $1 / 50 \mathrm{~cm}^{3}$. Il se forme par l'addition de l'acide un précipité insignifiant, qui en général, disparaît en quelques minutes. Quand le liquide continue d'être trouble, il est un peu difficile d'observer la couleur de l'indicateur. Dans ce cas, il faut filtrer à travers du gros papier avant l'observation. Se basant sur le résultatdu titrage,on peut calculer la quantité d'acide nécessaire pour l'ensemble des caillettes coupées.

J'ai supposé ci-dessus qu'en macérant avec des solutions de chlorure de sodium et d'acide borique, on obtient des extraits dont le $\mathbf{P}_{H}$ est $>4,80$. Dans la pratique, il arrive quelquefois, que l'acidité de ces extraits est telle, que le ferment est partiellement détérioré. En ee cas, on ne trouvera pas un minimum quand on opère suivant la méthode $a$; le temps de coagulation sera le plus court pour la solution la moins acide, et on doit contrôler le $\mathrm{P}_{\mathrm{H}}$ de cet extrait par voie potentio-ou colorimétrique. En suivant la méthode $b$, on aperçoit l'acidité trop élevée immédiatement par l'observation colorimétrique ; en ce cas, il faut ajouter prudemment une solution de borate de soude jusqu'à la couleur désirée.

Note IV. - L'acidité des présures qui doivent attendre dans.les magasins ou les fromageries. - Ici, cette acidité n'est pas aussi étroite que celle qu'on doit employer pour l'activation du zymogène à une température déterminée. La valeur du $\mathrm{P}_{\mathrm{H}}$ oscille entre 5,3 et 6,3 . La limite du côté alcalin a été fixée déjà par Van Dam (1) à l'aide de l'acide rosolique. On peut aussi employer le bleu de bromo-thymol ; cet indicateur, ajouté à une présure non diluée, doit montrer la teinte jaune. L'autre limite, du côté acide, est affirmée avec le rouge de méthyle. Cet indicateur doit montrer une couleur, qui est moins rouge que celle du mélange d'acélates no 4 (tableau VI). En diluant, au dixième, les présures avec de l'eau, si on y en ajoute un peu, la valeur de $\mathrm{P}_{\mathrm{H}}$ est peut-être augmentée par quelques unités de la dernière décimale. Il est donc recommandable de ne pas diluer avant l'épreuve colorimétrique, quand on examine le côté acide.

Les principes rapportés ci-dessus ont été appliqués dans une fabrique de présure en Hollande. On a trouvé que les manipulations a suivre sont simples et qu'il est possible de raecourcir notablement le processus de la fabrication, ce qui a comme conséquence une augmentation de la capacité utile de l'usine.

\title{
UN MALENTENDU
}

\section{A PROPOS DE LA. FORMULE DE FLEISGHMANN,}

\author{
par L. PANCHAUD,
}

Docteur ès-Sciences,

Chimiste au Laboratoire Cantonal d'analyses de Genève.

Presque tous les traités sur l'analyse du lait on des denrées alimentaires mentionnent la formule de FleischmanN :

$$
\mathrm{E}=1,2 \times \mathrm{G}+2,665 \times 100\left(\frac{\mathrm{D}-1}{\mathrm{D}}\right)
$$

(1)ERevue générale du Lait, VII. 514, (1909). 
qui permet de calculer l'extrait sec $\mathrm{E}$ du lait à partir de sa densité $\mathrm{D}$ à $1 \breve{~}^{n}$ et de sa teneur en matière grasse G.

Les uns (Martin : Laiterie) disent que la formule (1) exprime $\mathrm{E}$ et G en $\%$, sans spécifier s'il s'agit de gr. pour $100 \mathrm{gr}$. ou $100 \mathrm{~cm}^{3}$ de lait; les autres (Bordas et Touplain : La Laiterie), en gr. par litre (le second terme de la formule étant alors $2,665 \times 1.000\left(\frac{D-I}{D}\right)$; d'autres enfin (Pellerin : Guide de l'Expert-Chimiste), en gr. pour $100 \mathrm{gr}$. de lait.

Devant ces divergences, il semble utile de préciser (sans vouloir actuellement discuter la valeur intrinsèque de la formule de FlersCHMANN) que la formule (1) ne doit être utilisée que pour G exprimé en gr. pour $100 \mathrm{gr}$. et qu'elle donne $\mathrm{E}$ en gr. pour $100 \mathrm{gr}$. de lait.

Lorsqu'on rapporte, comme en France, les résultats de l'analyse au litre de lait, on commet une erreur en introduisant dans (1) les valeurs de $G$ en $\mathrm{gr}$. pour $100 \mathrm{~cm}^{3}$, et on obtient alors pour $\mathrm{E}$ un chiffre intermédiaire entre l'extrait de $100 \mathrm{gr}$. et celui de $100 \mathrm{~cm}^{3}$ de lait.

Si l'on veut se servir de G trouvé en grammes pour $100 \mathrm{~cm}^{3}$, il faut modifier la formule (1).

En effet, posons :

$$
\begin{aligned}
& \left.\begin{array}{l}
\mathrm{E} \text { : extrait....... } \\
\mathrm{G} \text { : matière grasse }
\end{array}\right\} \text { pour } 100 \mathrm{gr} \text {. de lait. } \\
& \mathrm{e}: \text { extrait........ }
\end{aligned}
$$

On aura :

$$
\mathrm{E}=\frac{\mathrm{e}}{\mathrm{D}} \quad \mathrm{G}=\frac{\mathrm{g}}{\mathrm{D}}
$$

Introduisant ces valeurs dans (I), on a

ou :

$$
\frac{\mathrm{e}}{\mathrm{D}}=1,2 \times \frac{\mathrm{g}}{\mathrm{D}}+2,665 \times \frac{100(\mathrm{D}-1)}{\mathrm{D}}
$$

$$
\mathrm{e}=1,2 \times \mathrm{g}+2,665 \times 100(\mathrm{D}-1)
$$

Cette formule rend les calculs plus simples que la formule (1); elle est identique à celle que l'on trouve dans les traités de Girard, Gérard et Bonn, bien qu elle soit donnée par ces auteurs sous une forme différente se prêtant mal au calcul, et qui est :

$$
\mathrm{e}=\frac{\mathrm{D}-1+0,00448 \times \mathrm{g}}{0,00378}
$$

En effet, la formule (II) donne, si on divise les 2 membres par 266,5

$$
\frac{\mathrm{e}}{266,5}=\frac{1.2-\times g}{266.5}+\mathrm{D}-1
$$


ou :

$$
0,00375 \mathrm{e}=0,004502 \times \mathrm{g}+\mathrm{D}-1
$$

ou enfin :

$$
\mathrm{e}=\frac{\mathrm{D}-1+0,0045 \times \mathrm{g}}{0.00375}
$$

Ces deux traités spécifient bien que $e$ et $g$ sont exprimés en gr. pour $100 \mathrm{~cm}$. Différents auteurs, Pierre, Steinmann, Drmicher. (voir Annales de Chimie analytique, 1904), ayant étudié la formule de Fleischmann ou proposé de nouvelles formules en utilisant les constantes de Fleischmann, ont constaté qu'ils obtenaient la même formule « à une très légère variante près » :

$$
\begin{gathered}
\text { Pierre : } g=0,84 \times \mathrm{e}-222(\mathrm{D} \cdot \mathrm{1}) \\
\text { Demichel : } g=0,88 \times \mathrm{e}-233 \times(\mathrm{D}-1)
\end{gathered}
$$

sans remarquer que cette \& très légère variante » provenait du fait que la formule de FleiscimanN :

$$
\mathrm{G}=0,833 \times \mathrm{E}-222 \times \frac{(\mathrm{D}-1)}{\mathrm{D}}
$$

à l'inverse des leurs, exprimait $G$ en gr. pour $100 \mathrm{gr}$. de lait.

Pour nous rendre compte des erreurs résultant de l'emploi erroné de la formule (1), prenons l'exemple cité par Bordas et Touplain :

$$
\mathrm{g}=30 \mathrm{gr} \text {. par litre ; } \mathrm{D} \text { à } 15^{\circ}=1,030 \text {. }
$$

Ces auteurs, appliquant la formule (I) à tort, trouvent $113 \mathrm{gr}, 6$ d'extrait. Il leur aurait fallu appliquer la formule (II) qui leur aurait donné $115 \mathrm{gr} ., 9$, soit une différence de plus de 2 grammes ( 2 gr.,4) par litre. Pour utiliser la formule (I) à bon escient, on aurait dù prendre pour $\mathrm{G}: \frac{3,0}{1,030}=2,9$ et on aurait obtenu $112 \mathrm{gr} ., 5$ d'extrait par kilo de lait. On vérifierait aisément que $142,5 \times 1,030=115,9$.

Bordas et Touplain donment (p. 108 de leur livre) une table poux le calcul de l'extrait see selon la formule (I); il faut y lire matière grasse pour $100 \mathrm{gr}$. au lieu de matière grasse pour $100 \mathrm{~cm}^{3}$. La même table se trouve dans Martin avec l'indication $\%$, sans dire de quel $\%$ il s'agit. Il doit ètre bien entendu que ces tables expriment l'extrait de $100 \mathrm{gr}$. de lait à partir de la matière grasse exprimée. elle aussi, pour $100 \mathrm{gr}$. de lail. Les erreurs résultant de l'emploi injustifié de la formule (I) sont d'autant plus élevées que la densité du lait et son taux en matière grasse sont plus forts.

Il est bon aussi de spécifier que le calcnlateur AckermanN (dont parlent ces traités), qui est une unachine à calculer résolvant l'équation (I), donne l'extrait en gr. pour $100 \mathrm{gr}$. de lait à partir de la matière grasse en gr. pour $100 \mathrm{gr}$. de lait. Si le calcula'eur perte 
les indications peu précises : graisse, extrait $\%$, cela tient, au fait qu'en Suisse et dans l'immense majorité des pays, on exprime les résultats en gr. pour $100 \mathrm{gr}$. de lait. En utilisant donc le chiffre de matière grasse pour $100 \mathrm{~cm}^{3}$, on commet avec le calculateur les mêmes erreurs dont il a été question plus haut. Ces constatations sont peutêtre de nature à expliquer en partie les différences observées entre l'extrait calculé, en se trompant avec la formule (I) ou le calculateur Ackermann, et l'extrait obtenu par pesée rapporté à $100 \mathrm{~cm}^{3}$ de lait.

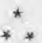

Il subsiste toujours une certaine équivoque au sujet du procédé de Gerber pour le dosage de la matière grasse. Du fait que l'on mesure $11 \mathrm{~cm}^{3}$ de lait, on pourrait déduire que l'échelle des butyromètres est graduée en gr. pour $100 \mathrm{~cm}^{3}$. C'est du moins l'opinion de tous les traités français que j'ai eu l'oceasion de parcourir.

Cependant, Gerber dans son opuscule : Analyse des produits laitiers, dit que " chaque degré de l'échelle indique $0,1 \%$ de matière grasse, valeur en poids "s, ce qui est évidemment peu net. FleisGhmanN est plus catégorique; il spécifie que les $\%$ sont rapportés au poids du lait.

Tous les auteurs de tous les pays, sauf la France, rapportent les résultats d'analyse à $100 \mathrm{gr}$. de lait, et lorsqu'ils parlent du dosage de la matière grasse du lait par les méthodes d'extraction (GotruiesRöse, Schmidt-Bondzrnsky...) divisent le poids de la matière grasse obtenue par le poids spécifique du lait, afin de le ramener à $100 \mathrm{gr}$.. quand le lait a été mesuré avec une pipette. Mais ils ne parlent jamais de cette opération lors du dosage, selon GERBER, preuve que le chiffre obtenu avec ce dernier procédé, doit, à leur avis, exprimer la matière grasse pour $100 \mathrm{gr}$, de lait. D'ailleurs certains auteurs, pour comparer les résultats du Gerber avec ceux des méthodes d'extraction, et afin d'éviter tout ealcul supplémentaire, mesurent $9 \mathrm{~cm} ., 7$, soit $10 \mathrm{gr}$. (si l'on admet une densité moyenne, du lait de 1,030); de la sorte, le résultat des méthodes d'extraction se trouve directement exprimé pour $100 \mathrm{gr}$., et il n'est ainsi pas nécessaire de faire intervenir la densité du lait dans le calcul.

Si l'on avait encore des dontes à ce sujet, on lirait avec fruit le travail de ReIss (Zeitschrift für Untersuchung, d. Nahr. u. g., Tome 36, pp. 273-78, 1918), dans lequel cet auteur cherche à élucider la question suivante : l'échelle des butyromètres est-elle calibrée au moyen de matière grasse du lait, ou de matière grasse telle qu'on l'obtient dans le procédé Gerber, par action de l'acide sulfurique et de l'alcool amylique sur le lait. La question devait être posée parce 
que Gerber avait toujours gardé une réserve prudente sur ce sujet. Rerss détermine à la température de $6{ }^{\circ}$ (à laquelle doit se faire la lecture des butyromètres) le poids spécifique de la matière grasse du lait, soit: 0,8827 , le poids de la matière grasse entre les points extrêmes de l'échelle butyrométrique $0-90$, soit: 1,026 g., et le volume intérieur du tube gradué entre ces deux divisions, soit : 1.1628 $\mathrm{em}^{3}$.

Ces données permettent de résoudre la question posée. Admettons qu'un lait de densité 1,033 ait donné au Gerber une matière grasse de $3 \%$ (30 divisions) Le volume entre ces 30 divisions est de $0.3876 \mathrm{~cm}^{3}$, ce qui exrrespond à un poids de matière grasse de lait de $342 \mathrm{mmg}$.

Si lon suppose que la graduation des butyromètres est établie en gr. pour $100 \mathrm{~cm}^{3}$ de lait, comme on est parti de $11 \mathrm{~cm}^{3}$ de lait, on aura done $330 \mathrm{mmg}$. de matière grasse de lait qui occuperaient un volume de $0,3793 \mathrm{~cm}^{3}$, soit 28,9 divisions. Mais si la graduation a été établie en $\mathrm{gr}$., pour $100 \mathrm{gr}$. de lait, pipetter $11 \mathrm{~cm}^{3}$ de lait équivant à peser 11,363 gr. de lait qui renferment $341 \mathrm{mmg}$. de matière grasse

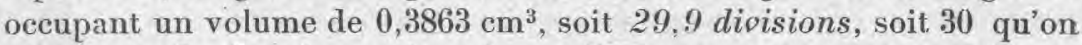
a lues. Le Gerber donne donc la matière grasse en gr., pour $100 \mathrm{gr}$. de lait.

En réalité, on a affaire dans le GERBER à une matière grasse assez dillérente de celle du lait, ra densité n'est plus que de 0,8523 , le poids de matiere grasse entre 90 divisions n'est que de $0,9918 \mathrm{gr}$. La méthode de Gerber est une méthode de compensation : à la place du polume théorique de matière grasse de lait, on lit exactement le mème volume de matière grasse Gerber.

Donc, si on utilise les résultats du Gerber avec la formule (1) ou avec le calculateur Ackermann, on obtient en réalité l'extrait en g'. pour $100 \mathrm{gr}$, de lait, ce dont il faut tenir compte si l'on compare avec: les résultats de la pesés de $10 \mathrm{~cm}^{3}$ de lait.

Conclusions. - $1^{\circ}$ Pour calculer l'extrait sec $e$ de $100 \mathrm{~cm}^{3}$ de lait à partir de la matière grasse $g$ de $100 \mathrm{~cm}^{3}$ de lait, on ne peut. utiliser la formule de Freischmann (I), il faut employer la formule modifiée :

$$
e=1,2 \times g+2,665 \times 100(\mathrm{D}-1)
$$

2. Lorsque l'on utilise la matière grasse obtenue selon GERBER, le calcul de lextrait sec ne peut se faire qu'à l'aide de la formule de Fleischmann (I) qui donne l'extrait sec de $100 \mathrm{gr}$. de lait. 